\title{
ANÁLISE DA VELOCIDADE DO AR EM LEITO DE JORRO OPERANDO COM MISTURAS DE AREIA E BIOMASSA
}

\author{
A. P. MARQUES ${ }^{1}$, K. M. BARCELOS ${ }^{2}$, P. S. ALMEIDA ${ }^{2}$, T. P. XAVIER ${ }^{1}$ e T. S. LIRA ${ }^{1,2}$ \\ ${ }^{1}$ Universidade Federal do Espírito Santo, Departamento de Engenharias e Tecnologias \\ ${ }^{2}$ Universidade Federal do Espírito Santo, Programa de Pós-Graduação em Energia \\ E-mail para contato: taisa.lira@ufes.br
}

\begin{abstract}
RESUMO - A substituição de fontes fósseis por renováveis vem crescendo com o passar dos anos por questões político-ambientais mais rígidas. Assim, surge a biomassa, que por ter baixo custo e ser, normalmente, resíduo de algum processo, a conversão em energia torna-se atraente. Dentre os tipos de conversão de biomassa, destaca-se a pirólise, que permite a formação de diversos produtos, a depender das condições operacionais. Como reator, indica-se o leito de jorro, que fornece boa mistura de partículas, ajudando na troca de calor entre as fases. Assim, com objetivo de estudar a velocidade do ar para misturas binárias de biomassa-areia, em um leito de jorro cônico, foram feitos ensaios com diferentes alturas de leito $(6,8$ e $10 \mathrm{~cm})$, frações mássicas de biomassa (cacau e macadâmia: $25,50,75 \%$, coco: $10,25,40 \%$ ) e abertura de cone de $45^{\circ}$ e $60^{\circ}$. Além disso, novos parâmetros para correlações empíricas de velocidade mínima de jorro existentes foram estimados.
\end{abstract}

\section{INTRODUÇÃO}

A conversão da biomassa para uma fonte energética pode ser feita através de processos como a combustão direta, de processos termoquímicos ou de processos biológicos (ANEEL, 2005). A pirólise é um processo que ocorre na ausência de oxigênio ou em quantidades muito pequenas, para realizar a degradação térmica do combustível sólido. Essa técnica é considerada a mais promissora, pois permite que seu processo seja direcionado para a produção de um ou outro produto, alterando as condições operacionais (SANTOS, 2011).

O bio-óleo, parte orgânica dos produtos da pirólise, pode ser usado como combustível, e a pirólise rápida, processo que favorece a formação deste produto, se destaca cada vez mais no cenário da conversão termoquímica de biomassa (BARCELOS, 2016).

O leito de jorro vem sendo estudado como reator para este tipo de processo por ser um equipamento que promove intensa mistura de partículas em seu interior, acarretando em elevados coeficientes de transferência de calor e massa (ALMEIDA, 2015). Segundo Rao (2000), em um leito fluidizado, a biomassa não pode ser tão facilmente fluidizada devido suas formas peculiares, tamanhos e densidades. Acredita-se que o leito de jorro atua de forma semelhante, então para auxiliar o processamento e a estabilidade jorro no reator, um segundo sólido inerte como areia, é usada. O inerte também auxilia na troca de calor no processo. 
Para possibilitar um regime de jorro estável alguns parâmetros específicos, como diâmetro de partícula, altura do leito estático, vazão do fluido de entrada e ângulo de abertura da base cônica devem ser avaliados (BÉTTEGA, 2009). Na literatura apresentam-se diversos estudos avaliando os efeitos destes parâmetros na vazão de mínimo jorro, trazendo equações empíricas para descrever a vazão de ar necessária ao regime estável. Muitos desses estudos se limitam a equações de monopartículas, como Olazar et al. (1992) que propuseram uma nova correlação, para ângulos acima de $45^{\circ}$, com erro menor que $8 \%$. A equação abrange diferentes sólidos, com diferentes diâmetros e geometrias, mas não permite a mistura destes. Bai et al. (1996), Asif (2010) e Paudel e Feng (2013) estudaram misturas de sólidos para predizer a velocidade do ar, inserindo um conceito de diâmetros e densidades efetivas, mas apenas para leitos fluidizados.

Então, com o objetivo de contribuir para a aplicação do leito de jorro cônico como um reator de pirólise para misturas de areia e biomassa, este trabalho teve o intuito de estudar a velocidade do ar em leito de jorro cônico submetido a diferentes condições operacionais, a fim de estimar parâmetros para as correlações empíricas de velocidade mínima de jorro.

\section{METODOLOGIA}

\subsection{Propriedades dos materiais}

As biomassas e areias utilizadas nos experimentos foram previamente caracterizadas, estando suas propriedades físicas dispostas nas Tabelas 1. Para as partículas de biomassa com diâmetro médio de $1,55 \mathrm{~mm}$, usou-se a areia grossa de $2,58 \mathrm{~mm}$ e para biomassas de diâmetro médio de 2,58mm, usou-se a areia fina.

Tabela 1 - Propriedades físicas das biomassas e areia.

\begin{tabular}{|c|c|c|c|}
\hline Propriedades & Densidade aparente, $\rho\left(\mathrm{kg} / \mathrm{m}^{3}\right)$ & Diâmetro médio $(\mathrm{mm})$ & Esfericidade, $\phi$ \\
\hline \hline Casca de macadâmia & 1190 & 1,55 & 0,72 \\
\hline Carpelo de macadâmia & 460 & 1,55 & 0,75 \\
\hline Casca de cacau & 879 & 2,58 & 0,53 \\
\hline Casca de coco & 478 & 2,58 & 0,60 \\
\hline Areia fina & 2644 & 1,55 & 0,79 \\
\hline Areia grossa & 2624 & 2,58 & 0,76 \\
\hline
\end{tabular}

\subsection{Unidade e Procedimento Experimental}

$\mathrm{Na}$ unidade experimental (representação esquemática na Figura 1) temos: 1) leito de jorro cônico-cilíndrico, 2) termopar, 3) transdutor de pressão da marca Dwyer, modelo 616C$4,4)$ aquecedor, 5) soprador centrífugo de 2HP IBRAM com um fluxo máximo de ar de $\left.4,5 \mathrm{~m}^{3} / \mathrm{min}, 6\right)$ condicionador de sinais, 7) placa de aquisição de dados A/D, da marca National Instruments e 8) computador.

Para a realização do experimento, as partículas de biomassa e areia foram pesadas e misturadas seguindo as proporções mássicas, até completarem as alturas de leito estático. Em seguida, para construção das curvas características, foi aumentada a velocidade do ar gradativamente até atingir regime instável, passando pela condição de jorro. Posteriormente, 
a velocidade do ar foi reduzida até a velocidade mínima alcançada pelo equipamento, e foram anotados os dados de queda de pressão correspondentes à cada velocidade do ar alcançada. Assim, foi possível determinar a velocidade de mínimo jorro, com auxílio do software LabView 7.0. O cálculo do desvio padrão da queda de pressão foi realizado para validar a estabilidade do leito. O controle da velocidade foi realizado com o auxílio de um termoanemômetro digital, da marca CE Instrutherm TAD-500, posicionado na saída de ar superior do equipamento. Os procedimentos ocorreram à uma mesma temperatura ambiente e ar injetado de $25^{\circ} \mathrm{C}$.

Figura 1 - Esquema da unidade experimental Fonte: Adaptado de Melo, 2014.

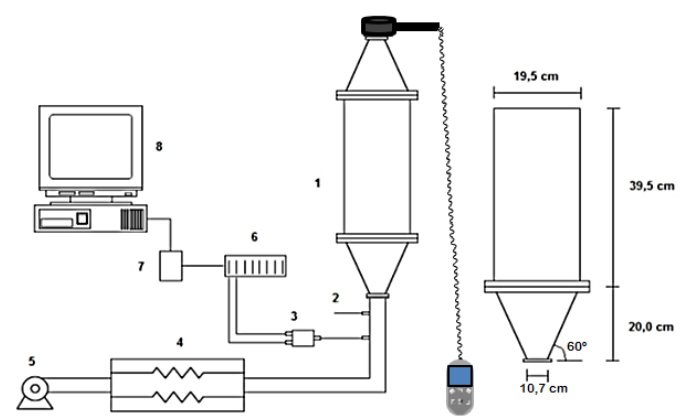

Segundo Barcelos (2016), para as alturas estudadas de 6,8 e $10 \mathrm{~cm}$, não ocorre jorro ao se operar com frações acima de $40 \%$ de casca de coco, pois não há quantidade de inerte suficiente para auxiliar a biomassa, levando à segregação de partículas. Desta forma, para este trabalho, foi limitado em 10, 25 e $40 \%$ de fração mássica de casca de coco, e para as demais biomassas, manteve-se 25,50 e $75 \%$ de fração mássica.

\subsection{Correlações empíricas para velocidade mínima de jorro}

A partir dos valores experimentais de velocidade mínima obtidos, e com o software MATLAB, os parâmetros para as correlações foram estimados, através de algoritmo de evolução diferencial. As correlações utilizadas estão dispostas na Tabela 2.

Tabela 2 - Correlações empíricas para predição da velocidade de mínimo jorro

\begin{tabular}{|c|c|}
\hline Autor & Correlação \\
\hline \hline Nikolaev e Golubev (1964) & $\operatorname{Re}_{\mathrm{ms}}=0,051 \mathrm{Ar}^{0,59}\left(\mathrm{D}_{\mathrm{i}} / \mathrm{D}_{\mathrm{c}}\right)^{0,1}\left(\mathrm{H}_{\mathrm{o}} / \mathrm{D}_{\mathrm{c}}\right)^{0,25}$ \\
\hline Tsvik et al. (1967) & $\operatorname{Re}_{\mathrm{msi}}=0,4 \mathrm{Ar}^{0,52}\left(\mathrm{H}_{\mathrm{o}} / \mathrm{D}_{\mathrm{i}}\right)^{1,24}[\tan (\mathrm{\gamma} / 2)]^{0,42}$ \\
\hline Goltsiker (1967) & $\operatorname{Re}_{\mathrm{msi}}=73 \mathrm{Ar}^{0,14}\left(\mathrm{H}_{\mathrm{o}} / \mathrm{D}_{\mathrm{i}}\right)^{0,9}\left(\rho_{\mathrm{p}} / \rho\right)^{0,47}$ \\
\hline Markowski e Kaminski (1983) & $\operatorname{Re}_{\mathrm{msi}}=0,028 \mathrm{Ar}^{0,57}\left(\mathrm{D}_{\mathrm{c}} / \mathrm{D}_{\mathrm{i}}\right)^{1,27}\left(\mathrm{H}_{\mathrm{o}} / \mathrm{D}_{\mathrm{i}}\right)^{0,48}$ \\
\hline Olazar et al. (1992) & $\operatorname{Re}_{\mathrm{msi}}=0,126 \operatorname{Ar}^{0,5}\left[\mathrm{D}_{\mathrm{b}} / \mathrm{D}_{\mathrm{i}}\right]^{1,68}[\tan (\mathrm{\gamma} / 2)]^{-0,57}$ \\
\hline
\end{tabular}

Nas quais a nomenclatura apresentada é definida como: Ar para o número de Arquimedes; $\mathrm{D}_{\mathrm{b}}$ diâmetro acima do leito estático $(\mathrm{m})$; $\mathrm{D}_{\mathrm{c}}$ diâmetro de coluna $(\mathrm{m})$; $\mathrm{D}_{\mathrm{i}}$ diâmetro de entrada do ar (m); $R_{\text {msi }}$ Número de Reynolds para velocidade mínima de jorro baseada no $\mathrm{D}_{\mathrm{i}} ; \gamma$ ângulo do cone (rad); $\rho$ densidade do fluido $\left(\mathrm{kg} / \mathrm{m}^{3}\right) ; \rho_{\mathrm{p}}$ densidade da partícula $\left(\mathrm{kg} / \mathrm{m}^{3}\right)$; $\mathrm{H}_{\mathrm{o}}$ altura de leito estático (m). 
Devido ao uso de mistura de areia com biomassa, foram calculados o diâmetro efetivo e a massa específica efetiva da mistura de partículas. Para isto, foi usada a definição de Asif (2010), que considera a fração volumétrica e a esfericidade das partículas, e a de Bai et al (1996), que considera a fração mássica. E para verificação dos resultados foram calculados os erros relativos dos parâmetros estimados em relação aos experimentais.

\section{RESULTADOS E DISCUSSÃO}

A partir da realização do procedimento experimental, puderam ser traçadas as curvas características para cada biomassa em cada fração e altura. Um exemplo de uma curva característica está representado na Figura 2.

Figura 2 - Curva característica para a casca do cacau para uma altura de $10 \mathrm{~cm}$ e fração mássica de biomassa de $25 \%$.

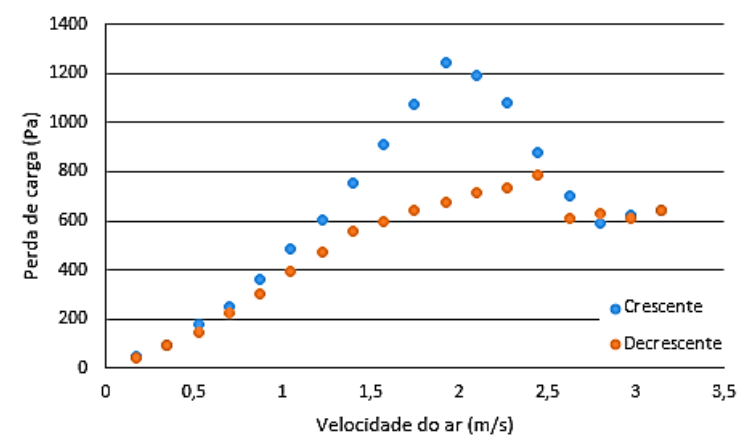

Todas as curvas obtidas apresentaram características pertinentes ao leito de jorro, passando por uma região de leito fixo, seguida de um estouro do jorro, até atingir um valor de mínimo, onde uma mudança na velocidade não causava alteração da perda de carga. Assim, iniciou-se o decréscimo da velocidade, passando pela velocidade de mínimo jorro, até o mínimo do equipamento.

A partir das curvas características, determinou-se a velocidade de mínimo jorro para todas as condições experimentais, como exemplificado na Tabela 3 para a casca de cacau. Nesta tabela temos os dados de altura de leito estático $\left(\mathrm{H}_{\mathrm{o}}\right)$, fração mássica de biomassa $\left(\mathrm{X}_{\mathrm{b}}\right)$, velocidade mínima de jorro $\left(\mathrm{V}_{\mathrm{mj}}\right)$, porosidade do leito de partículas $\left(\mathcal{E}_{\mathrm{lp}}\right)$, massa total do leito de partículas $\left(\mathrm{M}_{\mathrm{lp}}\right)$ e o ângulo de abertura do cone $(\mathrm{\gamma})$.

Tabela 3 - Dados experimentais e velocidade mínima de jorro para a casca de cacau em fração de $25 \%$ de biomassa

\begin{tabular}{|c|c|c|c|c|c|}
\hline$H_{o}(m)$ & $X_{b}$ & $V_{m j}(\mathrm{~m} / \mathrm{s})$ & $\mathcal{E}_{l p}$ & $M_{l p}(g)$ & $\gamma$ \\
\hline \hline 0,06 & 0,25 & 1,75 & 0,51 & 540,146 & $60^{\circ}$ \\
\hline 0,08 & 0,25 & 2,27 & 0,51 & 820,324 & $60^{\circ}$ \\
\hline 0,10 & 0,25 & 2,62 & 0,51 & 1100,33 & $60^{\circ}$ \\
\hline 0,06 & 0,25 & 6,68 & 0,51 & 200 & $45^{\circ}$ \\
\hline 0,08 & 0,25 & 7,84 & 0,51 & 300 & $45^{\circ}$ \\
\hline 0,10 & 0,25 & 9,29 & 0,51 & 440 & $45^{\circ}$ \\
\hline
\end{tabular}


Pode-se perceber que os valores de velocidade mínima de jorro aumentam conforme o leito cresce, pois há um aumento da massa total do leito, e diminuem com o aumento do ângulo, devido a diminuição da componente da força peso na direção da força de gravidade.

Com estes valores, estimou-se novos parâmetros para as correlações empíricas da Tabela 4. Observa-se que tanto a correlação de Tsvik et al. (1967) quanto de Olazar et al. (1992) apresentaram erros relativos similares e menores que os demais, quando utilizada a definição de Asif (2010) para diâmetro e densidade efetivos. Este fato pode ser explicado pela consideração do efeito da geometria da partícula devido ao termo da esfericidade.

Pela Tabela 4, ainda pode ser observado que as correlações nas quais menores erros foram encontrados, são as que consideram o ângulo de abertura do cone, Olazar et al. (1992) e Tsvik et al. (1967). A presença de maiores erros nas demais equações, se deve ao fato de não considerar esse ângulo de abertura, já que os valores de velocidade mínima de jorro para os ângulos de $45^{\circ}$ e $60^{\circ}$ diferem bastante em seus módulos.

Tabela 4 - Parâmetros estimados e erros relativos para as correlações empíricas.

\begin{tabular}{|c|c|c|c|c|c|c|}
\hline \multirow{2}{*}{ Correlação } & \multirow{2}{*}{$\begin{array}{c}\text { Definição da propriedade } \\
\text { efetiva }\end{array}$} & \multicolumn{4}{|c|}{ Parâmetros } & \multirow{2}{*}{$\begin{array}{l}\text { Desvios } \\
\text { Relativos }\end{array}$} \\
\hline & & $\mathrm{K}_{1}$ & $\mathrm{~K}_{2}$ & $\mathrm{~K}_{3}$ & $\mathrm{~K}_{4}$ & \\
\hline \multirow{2}{*}{ Olazar et al. (1992) } & Asif(2010) & $1,1,996$ & 0,484 & 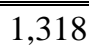 & 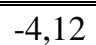 & $\frac{13,68 \%}{13 \%}$ \\
\hline & Bai et al. (1996). & 1,227 & 0,513 & 1,556 & $-3,48$ & $26,73 \%$ \\
\hline \multirow{2}{*}{ Tsvik et al. (1967) } & Asif (2010) & 6,958 & 0,437 & 0,755 & $-4,23$ & $13,62 \%$ \\
\hline & Bai et al. (1996). & 1,869 & 0,515 & 0,131 & $-3,62$ & $35,00 \%$ \\
\hline \multirow{2}{*}{ Goltsiker (1967) } & Asif (2010) & 145,8 & 0,189 & 0,756 & 0,427 & $42,87 \%$ \\
\hline & Bai et al. (1996). & 631,8 & $-0,20$ & 0,653 & 0,921 & $40,18 \%$ \\
\hline \multirow{2}{*}{$\begin{array}{c}\text { Markowski e Kaminski } \\
\text { (1983) }\end{array}$} & Asif (2010) & 0,194 & 0,428 & 0,756 & 9,583 & $43,32 \%$ \\
\hline & Bai et al. (1996). & 0,213 & 0,382 & 0,741 & 9,644 & $46,21 \%$ \\
\hline \multirow{2}{*}{ Nikolaev e Golubev (1964) } & Asif (2010) & 850,8 & 0,428 & 0,876 & 0,756 & $32,76 \%$ \\
\hline & Bai et al. (1996). & 536,0 & 0,430 & 1,168 & 0,682 & $42,57 \%$ \\
\hline
\end{tabular}

Assim, pode-se reescrever as correlações de Olazar et al. (1992) e Tsvik et al. (1967), de menor erro, segundo as equações 1 e 2 , respectivamente.

$$
\begin{aligned}
& \operatorname{Re}_{m s i}=1,996 \mathrm{Ar}^{0,484}\left[D_{\mathrm{b}} / \mathrm{D}_{\mathrm{i}}\right]^{1,318}[\tan (\gamma / 2)]^{-4,12} \\
& \operatorname{Re}_{\mathrm{msi}}=6,958 \mathrm{Ar}^{0,437}\left(\mathrm{H}_{\mathrm{o}} / \mathrm{D}_{\mathrm{i}}\right)^{0,755}[\tan (\gamma / 2)]^{-4,23}
\end{aligned}
$$

Através das novas equações calculou-se os novos valores de velocidade mínima de jorro para as biomassas, nos quais foram encontrados para Olazar et al. (1992), por exemplo, para casca de cacau em $25 \%$ em todas alturas, 1,$4351 ; 1,7134$ e $2,1440 \mathrm{~m} / \mathrm{s}$ para o ângulo de $60^{\circ} \mathrm{e}$ 5,6370; 6,7301 e 8,4215 m/s para 45 ${ }^{\circ}$. E para equação de Tsvik et al. (1967), para o mesmo exemplo, 1,3923; 1,7300 e $2,0475 \mathrm{~m} / \mathrm{s}$ para o ângulo de $60^{\circ}$ e 5,$6722 ; 7,0483$ e $8,3416 \mathrm{~m} / \mathrm{s}$ para o ângulo de $45^{\circ}$.

\section{CONCLUSÃO}

Novos parâmetros para as correlações empíricas da literatura foram estimados para predizer a velocidade de mínimo jorro de misturas de biomassa e areia. A definição de Asif (2010) para diâmetro e densidade efetivos foi a mais adequada, por ponderar a geometria da partícula, e as equações de Olazar et al. (1992) e Tsvik et al. (1967) foram as que 
apresentaram menores erros relativos, por considerarem os diferentes ângulos de abertura do cone. As demais correlações apresentaram erros relativos altos, devido à grande diversidade de dados experimentais.

\section{REFERENCIAS}

AGÊNCIA NACIONAL DE ENERGIA ELÉTRICA. Atlas de energia elétrica do Brasil. 2 ed., cap. 5, p.77-92 Brasília: ANEEL 2005. ISBN 85-87491-09-1.

ALMEIDA, P. de S. Contribuições para a pirólise da casca de cacau em leito de jorro - Estudos da fluidodinâmica experimental e simulação via CFD. 2015. 73 f. Dissertação (Mestrado em Energia) Programa de Pós-Graduação em Energia, Universidade Federal do Espírito Santo (UFES), São Mateus, 2015.

ASIF, M. Minimum Fluidization Velocities of Binary-Solid Mixtures: Model Comparison. International Journal of Chemical, Molecular, Nuclear, Materials and Metallurgical Engineering, v.4, n.3, 2010.

BAI, D.; MASUDA, Y.; NAKAGAWA, N.; KATO, K. Hydrodynamic behavior of a binary solids fluidized bed. Journal of Chemical Engineering of Japan, v. 29, p. 211-216, 1996.

BARCELOS, K. M. Análise do potencial do leito de jorro como reator para pirólise da casca de coco: estudo experimental e simulação via CFD. 2016. 114 f. Dissertação (Mestrado em Energia) - Programa de Pósgraduação em Energia, Universidade Federal do Espírito Santo (UFES), São Mateus, 2016.

BÉTTEGA, R. Contribuições ao estudo da fluidodinâmica em leitos de jorro utilizando-se CFD. 2009. $154 \mathrm{f}$. Tese (Doutorado em Engenharia Química) - Programa de Pós-graduação em Engenharia Química, Universidade Federal de São Carlos (UFSCar), São Carlos, 2009.

EPSTEIN, N.; GRACE, J. R. Spouted and Spout-Fluid Beds: Fundamentals and Applications. 1 ed, 360 p., Nova Iorque: Cambridge University. 2011. ISBN 978-0-521-51797-3.

MARKOWSKI, A..; KAMINSKI, W. Hydrodynamic Characteristics of Jet Spouted Beds. Can. J. Chem. Eng. 1983,61,377-381.

MELO, J. L. Z. Análise do leito de jorro como sistema de contato para pirólise de compósito PEBD/AL. 2014. 72 f. Dissertação (Mestrado em Energia) - Programa de Pós-graduação em Energia, Universidade Federal do Espírito Santo (UFES), São Mateus, 2014.

NIKOLAEV, A. M..; GOLUBEV, L. G. Basic Hydrodynamic Charaderiatics of the Spouting Bed. Zzv. Vyssh. Ucheb. Zaved. Khim. Khim. Tekhnol. 1964, 7, 855.

OLAZAR, M.; JOSÉ, M. J. S.; AGUAYO, A. T.; ARANDES, J. M.; BILBAO, J. Stable Operation Conditions for Gas-Solid Contact Regimes in Conical Spouted Beds. Ind. Eng. Chem. Res., v.31, n.7, p. 1784-1792, Espanha, 1992.

RAO, T. R., BHEEMARASETTI, J. V. R., Minimum fluidization velocities of mixtures of biomass and sands. Energy. Nova Déli, v.26, p. 633-644, 2000.

SANTOS, K. G. dos. Aspectos fundamentais da pirólise de biomassa em leito de jorro: fluidodinâmica e cinética do processo. 2011. Tese (Doutorado em Engenharia Química) - Programa de Pós-Graduação em Engenharia Química, Universidade Federal de Uberlândia (UFU), Uberlândia, 2011.

TSVIK, M. Z.; NABIEV, M. N.; RIEV, N. U.; MERENKOV, K. V.; VYZGO, V. S. The Velocity for External Spouting in Then Combined Prowas for Production of Granulated Fertilizer. Uzb. Khim. Zh. 1967, 21 (2), 50. 